\title{
MATERIAL PARAMETERS CHARACTERIZING THE PERFORMANCE OF FLASHLAMP PUMPED $\mathrm{BaLa}_{1-x} \mathrm{Nd}_{x} \mathrm{Ga}_{3} \mathrm{O}_{7}$ LASER*
}

\author{
W. RyBa-Romanowski, S. GoląB \\ Institute of Low Temperature and Structure Research, Polish Academy of Sciences, \\ pl. Katedralny 1, 50-950 Wrocław, Poland
}

AND M. BERKOWSKI

Institute of Physics, Polish Academy of Sciences, Warszawa, Poland

(Received May 14, 1990)

\begin{abstract}
Physical properties of optically uniaxial $\mathrm{BaLa}_{1-x} \mathrm{Nd}_{x} \mathrm{Ga}_{3} \mathrm{O}_{7}$ crystals have been investigated. Specific heat, thermal expansion coefficients, thermal diffusivity, Young modules and Poisson ratios of the crystal have been determined. Basing upon these parameters and the available spectral data the operating conditions for lasers employing rods made of $\mathrm{BaLa}_{1-x} \mathrm{Nd}_{x} \mathrm{Ga}_{3} \mathrm{O}_{7}$ have been defined.
\end{abstract}

PACS numbers: 42.60.-b

\section{Introduction}

Complex oxides whose composition may be expressed by the general formula $\mathrm{ABC}_{3} \mathrm{O}_{7}$, where $\mathrm{A}$ stands for $\mathrm{Ca}, \mathrm{Sr}$ or $\mathrm{Ba}, \mathrm{B}$ stands for $\mathrm{La}$ or lanthanides from $\mathrm{La}$ to $\mathrm{Gd}$ and $\mathrm{C}$ for $\mathrm{Al}$ or $\mathrm{Ga}$, form tetragonal crystals with a gehlenite structure (space group $\mathrm{P} 42_{1} \mathrm{~m}$ ). Large single crystals of $\mathrm{BaLa}_{1-x} \mathrm{Nd}_{x} \mathrm{Ga}_{3} \mathrm{O}_{7}$ can be grown by the Czochralski method using the automatic control of crystals diameter [1]. Their optical and spectral properties have been investigated and reported in several papers [2-4]. Basing on the results of spectroscopic investigations and on preliminary laser experiments, it has been concluded that $\mathrm{BaLa}_{1-x} \mathrm{Nd}_{x} \mathrm{Ga}_{3} \mathrm{O}_{7}$ is of interest for practical application as a laser active material [3].

*This work was supported by the CPBR 8.14. 
One of the most important spectroscopic features of the crystal is the strong inhomogeneous broadening of spectral lines associated with transitions between electronic levels of the $4 f^{3}$ configuration of neodymium. The bandwidth of luminescence associated with the laser transition is about $160 \mathrm{~cm}^{-1}$ at room temperature, the stimulated emission cross section equals $2.7 \times 10^{-20} \mathrm{~cm}^{2}$ for the light polarized parallel to the optical axis and $8.8 \times 10^{-20} \mathrm{~cm}^{2}$ for the light polarized perpendicular to the optical axis of the crystals. The latter value is roughly ten times lower than the stimulated emission cross section of Nd:YAG. Obviously, the crystal studied is an example of an intermediate gain laser material which is not a serious contender in the design of CW lasers. On the other hand the broad luminescence band is an advantageous feature when an active material is employed in pulsed high energy lasers since the energy storage is inversely proportional to the stimulated emission cross section. Taking into account other advantages as polarized output and the possibility of varying gain by changing the rod axis one can conclude that $\mathrm{BaLa}_{1-x} \mathrm{Nd}_{x} \mathrm{Ga}_{3} \mathrm{O}_{7}$ is suitable for practical application in repetitively pulsed lasers pumped by conventional flashlamps or light emitting diodes.

The optical pumping generates an appreciable quantity of heat which gives rise to nonuniform temperature distribution within an active rod. The thermal effects obtained strongly influence the output beam quality and introduce considerable mechanical stresses in the laser rod.

The purpose of this work is the investigation of effects associated with absorption of the pumping light emitted by pulsed xenon lamps and the evaluation of material parameters useful in the design of lasers employing $\mathrm{BaLa}_{1-x} \mathrm{Nd}_{x} \mathrm{Ga}_{3} \mathrm{O}_{7}$.

4

\section{Results and discussion}

Spectral distribution of the pumping light emitted by conventional flashlamps is such that there is considerable absorption by host in ultraviolet and infrared region. Absorbed energy is converted to heat and frequently leads to formation of colour centres. The ultra violet absorption edge of $\mathrm{BaLaGa}_{3} \mathrm{O}_{7}$ is situated at about $40000 \mathrm{~cm}^{-1}$. However, a broad absorption band in the spectral region $30000-40000 \mathrm{~cm}^{-1}$ has been observed and attributed to defects due to deviations from stoichiometry [2]. We observed that absorption of light in this spectral region results in the strong green coloration of the crystal and drastic reduction of emitted laser energy. In Fig. 1 we present a partial absorption spectrum of $\mathrm{BaLa}_{0.99} \mathrm{Nd}_{0.01} \mathrm{Ga}_{3} \mathrm{O}_{7}$ recorded before and after irradiation by intense light emitted by a flashlamp with an envelope made of pure silica. Colour centres decay slowly and it takes few days to restore the initial condition. In order to avoid the ultraviolet absorption and formation of colour centres, commercially available flashlamps made of doped quartz should be used. Alternatively, adequate filtering can be obtained by introduction of a layer of pyrex glass between the lamp and the rod.

Thermal effects in repetitively pulsed laser rods depend on repetition rate and physical parameters of laser material. The thermal time constant $\tau$ of the rod 


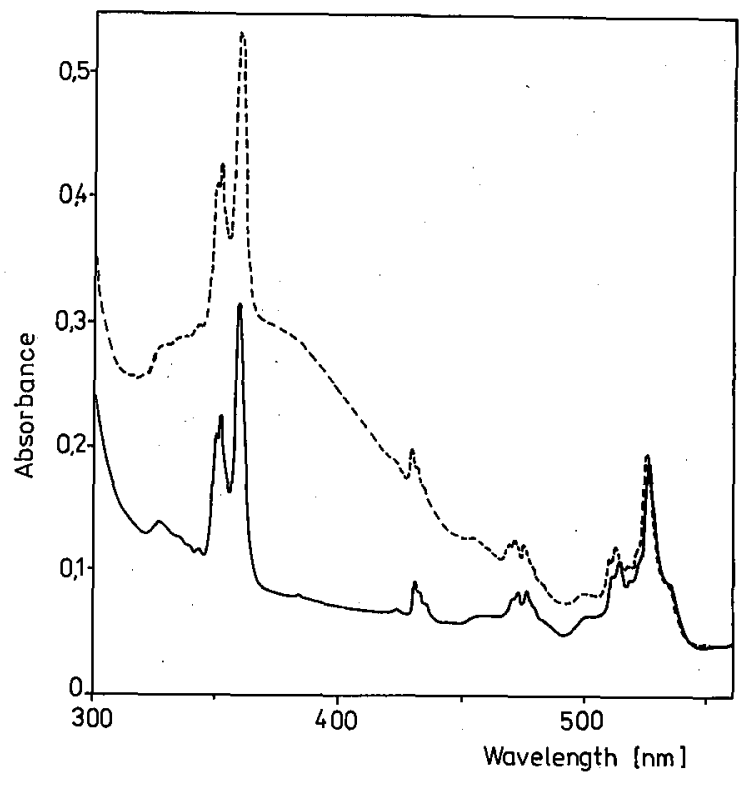

Fig. 1. Partial absorption spectrum of $\mathrm{BaLa}_{0.99} \mathrm{Nd}_{0.01} \mathrm{Ga}_{3} \mathrm{O}_{7}$ recorded before irradiation (solid line) and after irradiation (dotted line) by intense ultraviolet radiation in the $30000-40000 \mathrm{~cm}^{-1}$ spectral region. Sample thickness $5 \mathrm{~mm}$.

is defined as [5]:

$$
\tau=\frac{r_{0}^{2}}{k}
$$

where $r_{0}$ is the rod radius and $k$ is the thermal diffusivity related to material parameters by

$$
k=\frac{K}{c_{p} \rho},
$$

where $K$ is thermal conductivity, $c_{p}$ is specific heat and $\rho$ is mass density. When repetition intervals are long compared to the thermal time constant, an approximation of a single shot operation may be used. In this approximation the thermal gradients result solely from nonuniform absorption of the pumping light since the heat transport during short pumping pulse may be neglected. In order to evaluate the value of the thermal diffusivity the dependence of $c_{p}$ on temperature has been measured in the temperature range of $330-550 \mathrm{~K}$. The results of the measurements are shown in Fig. 2. Introducing into Eq. (2) the values $c_{p}=0.31 \mathrm{~J} / \mathrm{gK}$ (at 330 $\mathrm{K}), \rho=5.45 \mathrm{gcm}^{-3}$ and $K=0.11 \mathrm{~W} / \mathrm{cmK}$ taken from [3], we obtain a thermal diffusivity of $k=0.065 \mathrm{~cm}^{2} / \mathrm{s}$. The thermal time constant of the rod with a typical diameter $2 r_{0}=0.63 \mathrm{~cm}$ made of $\mathrm{BaLa}_{1-x} \mathrm{Nd}_{x} \mathrm{Ga}_{3} \mathrm{O}_{7}$ is $1.52 \mathrm{~s}$ at $330 \mathrm{~K}$ and it will slightly increase with increasing temperature. For comparison, the thermal 




Fig. 2. Dependence of specific heat $c_{p}$ of $\mathrm{BaLa}_{0.95} \mathrm{Nd}_{0.05} \mathrm{Ga}_{3} \mathrm{O}_{7}$ on temperature.

time constant for the Nd:YAG rod of the same diameter is $2.1 \mathrm{~s}$. The calculations have been tested experimentally by operating a small laser employing the $0.63 \mathrm{~cm}$ diameter $\mathrm{BaLa}_{0.95} \mathrm{Nd}_{0.05} \mathrm{Ga}_{3} \mathrm{O}_{7}$ rod pumped by a single linear flashlamp. At repetition intervals of $4 \mathrm{~s}$ the output beam parameters for consecutive shots monitored during an hour, were essentially the same as those observed at the first shot.

When repetition intervals are short compared to the thermal time constant the steady state condition will be reached in which the strong thermal gradient between the hot rod axis and relatively cool rod surface exists. The thermal gradient generates mechanical stresses in the material and optical distortions such as thermal lensing or thermally induced birefringence. It has been found [6] that the temperature distribution along the rod radius follows a parabolic function and the resulting tangential and axial stresses are particularly important at the centre and at the surface of the laser rod. In the case of isotropic material the thermally induced stress $\sigma$ at the surface of the laser rod can be expressed by the formula:

$$
\sigma=\frac{2^{1 / 2} \alpha E}{8 K(1-\nu)} \frac{P}{L}
$$

where $\alpha$ is the thermal coefficient of expansion, $E$ is Young's modulus, $K$ is thermal conductivity, $:$ is Poisson's ratio, $P$ is total heat dissipated in the rod and $L$ is the length of the rod. With increasing pumping power the tension on the rod surface can exceed the tensile strength of the rod. The maximum permissible pumping level may be estimated provided the material parameters are known. The thermal coeficient of expansion have been determined experimentally in the temperature range of $80-400 \mathrm{~K}$ for direction perpendicular $(\perp)$ and parallel $(\|)$ to the optical axis of $\mathrm{BaLa}_{0.95} \mathrm{Nd}_{0.05} \mathrm{Ga}_{3} \mathrm{O}_{7}$ crystal. The results of measurements are shown in Fig. 3. The anisotropy of the thermal coefficient of expansion is very small, particularly at room temperature, where $\alpha_{\perp}=12.82 \times 10^{-6} \mathrm{~K}^{-1}$ and $\alpha_{\|}=12.90 \times 10^{-6} \mathrm{~K}^{-1}$. The remaining mechanical constants in (3) can be evaluated using the elastic constants $c_{i k}^{E}$ determined from the measured parameters of thin-plate resonators [7]. Since the crystal investigated is of tetragonal symmetry 

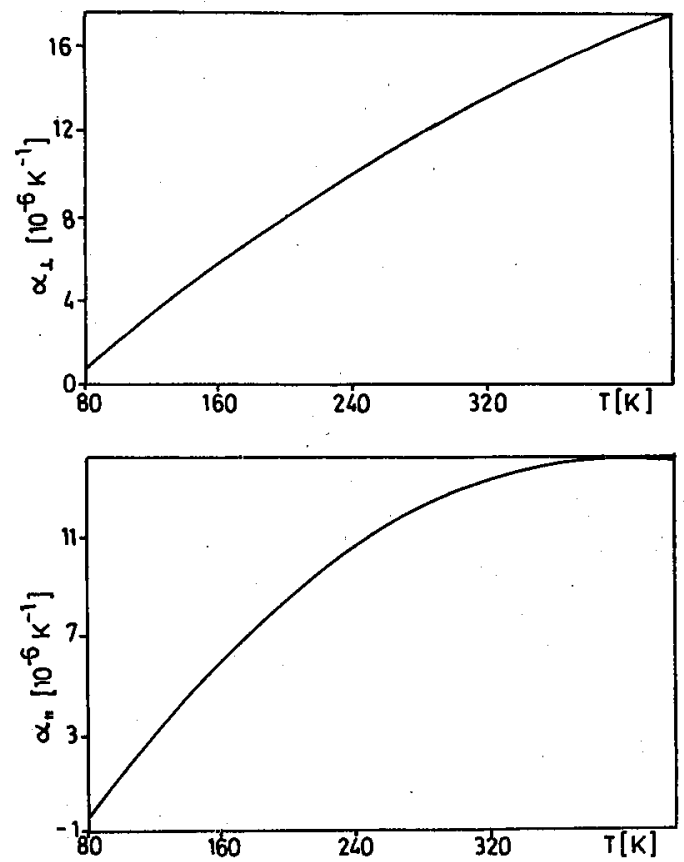

Fig. 3. Dependence of thermal expansion coefficient $\alpha$ on temperature for direction perpendicular to optical axis (upper) and for direction parallel to optical axis (lower) of $\mathrm{BaLa}_{0.95} \mathrm{Nd}_{0.05} \mathrm{Ga}_{3} \mathrm{O}_{7}$.

there are six independent elastic constant describing the behaviour of the material. Elastic compliances $s_{i k}^{E}$ have been calculated according to the relation [8]:

$$
s_{i k}^{E}=(-1)^{i+k} \frac{\Delta_{i k}^{c}}{\Delta^{c}}
$$

where $\Delta^{c}$ is the determinant of the constants $c_{i k}^{E}$ and $\Delta_{i k}^{c}$ is the minor determinant of $\Delta^{c}$ obtained by deleting the $i$-th line and $k$-th column. The obtained values are: $s_{11}=1.00 \times 10^{-11} \mathrm{~m}^{2} / \mathrm{N}, s_{12}=-0.33 \times 10^{-11} \mathrm{~m}^{2} / \mathrm{N}, s_{13}=-0.51 \times 10^{-11}$ $\mathrm{m}^{2} / \mathrm{N}, s_{33}=1.62 \times 10^{-11} \mathrm{~m}^{2} / \mathrm{N}$. Having the elastic compliances calculated we get two Young modules: $E_{11}=\left(s_{11}^{E}\right)^{-1}=1.00 \times 10^{11} \mathrm{~N} / \mathrm{m}^{2}, E_{33}=\left(s_{33}^{E}\right)^{-1}=0.62 \times$ $10^{11} \mathrm{~N} / \mathrm{m}^{2}$ and three Poisson ratios: $\nu_{12}=-s_{12}^{E}\left(s_{11}^{E}\right)^{-1}=0.33, \nu_{13}=-s_{13}^{E}\left(s_{33}^{E}\right)^{-1}=$ 0.31 and $\nu_{31}=-s_{13}^{E}\left(s_{11}^{E}\right)=0.51$.

In principle, having these parameters the thermally induced stresses may be calculated for any direction in an anisotropic crystal. It should be noted that in contrast to isotropic crystals like YAG, the thermally induced stresses in $\mathrm{BaLa}_{1-x} \mathrm{Nd}_{x} \mathrm{Ga}_{3} \mathrm{O}_{7}$ cause a slight modification of the uniform optical anisotropy, therefore the effects of thermal birefringence are reduced to negligible levels.

The presented data indicate that single crystals of $\mathrm{BaLa}_{1-x} \mathrm{Nd}_{x} \mathrm{Ga}_{3} \mathrm{O}_{7}$ are 
well suited for applications in repetitively pulsed, flashlamp pumped lasers, provided the UV free flashlamps are employed.

\section{References}

[1] W. Piekarczyk, M. Berkowski, G. Jasiołek, W. Ryba-Romanowski, M. Chabiera, Patent PRL No. 260309 (1986); US Patent No. 4, 820, 445 (1989).

[2] M. Berkowski, M.T. Borowiec, K. Pataj, W. Piekarczyk, W. Wardzyński, Physica $B$ 123, 215 (1984).

[3] W. Ryba-Romanowski, B. Jeżowska-Trzebiatowska, W. Piekarczyk, M. Berkowski, J. Phys. Chem. Solids 49, 199 (1988).

[4] W. Ryba-Romanowski, S. Goląb, J. Hanuza, M. Berkowski, J. Phys. Chem. Solids 50, 685 (1989).

[5] W. Koechner, Solid Stale Laser Engineering, Springer-Verlag, New York, Heidelberg, Berlin 1976.

[6] W. Koechner, Appl. Opt. 9, 1429 (1970).

[7] W. Soluch, R. Księżopolski, W. Piekarczyk, M. Berkowski, M. Goodberlet, J.F. Vetelino, J. Appl. Phys. 58, 2285 (1985).

[8] J.F. Nye, Physical Properties of Crystals, Their Representation by Tensors and Matrices, Clarendon Press, Oxford 1957. 